\title{
Obstacle-induced Branching in Filamentous Fungi
}

\author{
B. Gonzalez, P. Shange, R. La Mascus, G. Solis, D.P. Baluch and R.W. Roberson*
}

Arizona State University, Tempe, Arizona, United States

*Corresponding author: robby2@asu.edu

Filamentous fungi have impactful roles as primary and secondary decomposers in ecosystems, in symbiotic relationships with plant root structures, and as plant and animal pathogens [1]. Crucial to their ecological roles is the mechanisms they have evolved to navigate the difficult and varied substrates that they grow in. Fungi exhibit polarized growth, which is a balance of exocytosis and endocytosis, with exocytosis occurring at the apex of a growing fungal hypha and endocytosis occurring along an endocytic collar just below the apex [2]. When filamentous fungi impact an obstacle to their growth, they exhibit branching responses in attempts to navigate the obstacle. Obstacle-induced branching responses have been investigated in Neurospora crassa, a fungus belonging to the phylum Ascomycota, with previous research showing that impact angles greater than $35^{\circ}$ result in dichotomous branching [3]. These obstacle-induced branching responses have not been investigated in Rhizopus oryzae, a fungus belonging to the phylum Mucoromycota. Neurospora crassa and $R$. oryzae are evolutionarily divergent and employ different vesicular organizations at their hyphal tips to facilitate polarized growth and navigation $[4,5]$. Neurospora crassa has a Spitzenkörper (Spk), which is a tight organization of vesicles that act as a vesicle supply center and play some role in the direction of growth [6]. Rhizopus oryzae has a looser organization of vesicles at the apical tip known as the apical vesicle crescent (AVC) that facilitates exocytosis [7]. This research investigates the obstacle-induced branching responses of these fungi in order to compare their growth and navigation strategies. In addition, this work seeks to determine any evolutionary advantages offered to $N$. crassa by the Spk or R. oryzae by the AVC.

To investigate obstacle-induced branching responses, time-lapsed videos of growing hyphae were captured with phase contrast light microscopy using an EVOS-FL auto imaging system (ThermoFisher Scientific, Waltham, MA). Growth obstacles in the chambers were $10 \mu \mathrm{m}$ in height and composed of four sets of three different maze patterns (diamond, square, chevron) arranged radially around a central inoculation port. These patterns were chosen to provide a variety of obstacles to the hyphal growth of the fungi. Videos were analyzed using ImageJ-Fiji (NIH, Bethesda, MD) and Adobe Photoshop (Adobe Systems Inc., San Jose, CA) in order to analyze the branching characteristics, and create image plates.

Neurospora crassa and $R$. oryzae exhibited dichotomous branching when navigating the square mazes. When navigating the diamond mazes $R$. oryzae exhibited sub-apical branching. Neurospora crassa displayed dichotomous branching within the diamond mazes. Neurospora crassa displayed a hyperbranching response when encountering obstacles in the chevron mazes, resulting in a branching cascade (Fig. 1). Rhizopus oryzae also showed a higher degree of branching in the chevron mazes, but there were fewer branches formed over all (Fig. 2).

Neurospora crassa had different obstacle-induced branching responses than $R$. oryzae. In the chevron mazes, $N$. crassa displayed a hyper-branching response which resulted in a branching cascade that ultimately was unable to overcome the obstacle. This cascade represents a large investment of resources for $N$. crassa that fails to navigate the obstacle. The hyper-branching $N$. crassa displayed resulted in the halt of exploration of the maze chamber before completion. Rhizopus oryzae did exhibit more branch formations in the chevron mazes, however the sub-apical nature of the branches cannot be parsed from the normal branching of growing hyphae. Rhizopus oryzae's branching responses did not impede it from 
completing the mazes. In order to determine if sub-apical branching is in response to impacts with obstacles, and to characterize the hyper-branching responses, statistical analysis of branching distances and the quantity of branch formation is on-going.

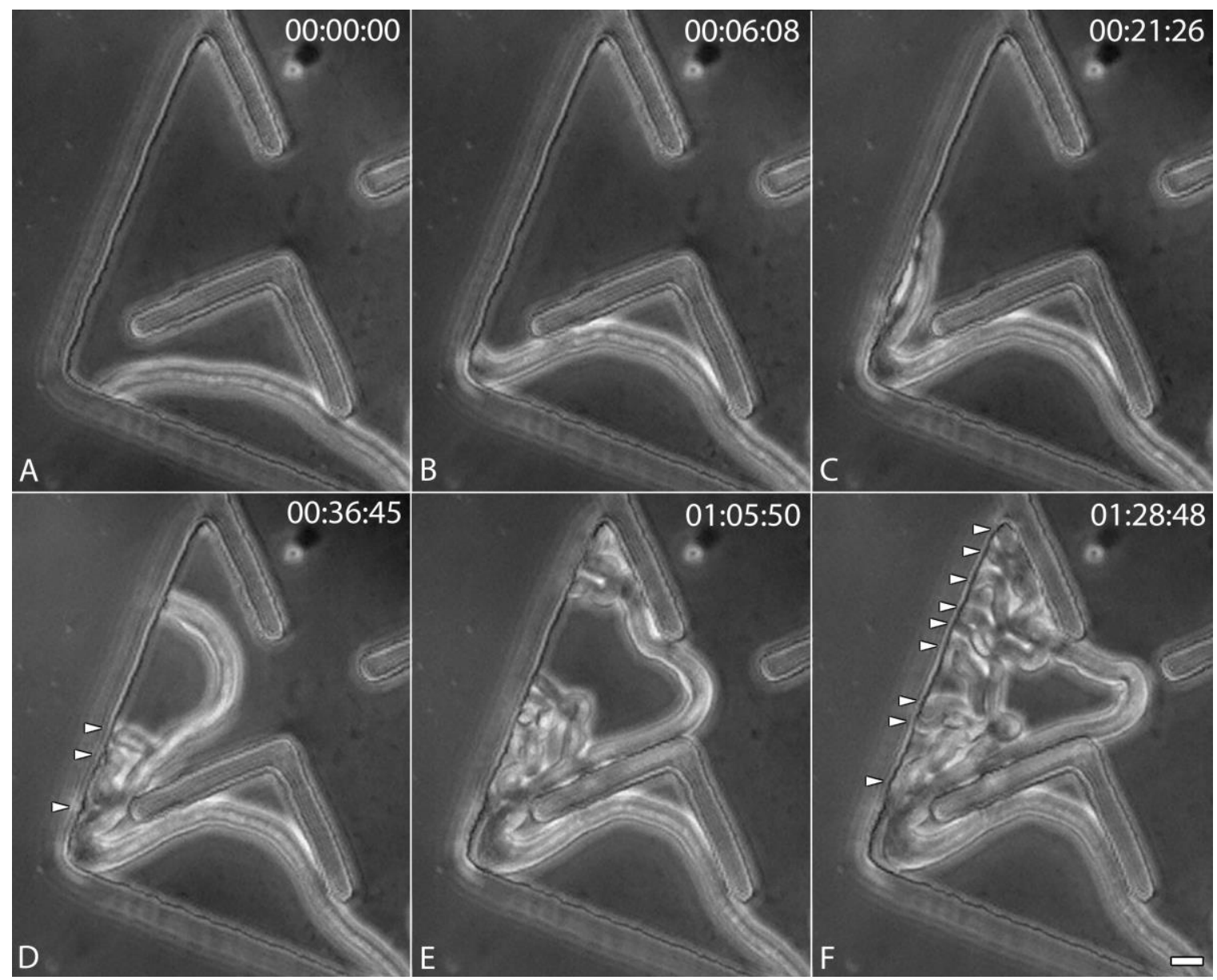

Figure 1. Neurospora crassa is seen approaching the maze edge (Fig. A) and being bent toward a new direction of growth (Figs. B, C). The hypha then begins to branch along several points, with each branch resulting in several new branches in a cascade (Figs. D, E, F). Scale bar $=10 \mu \mathrm{m}$. 


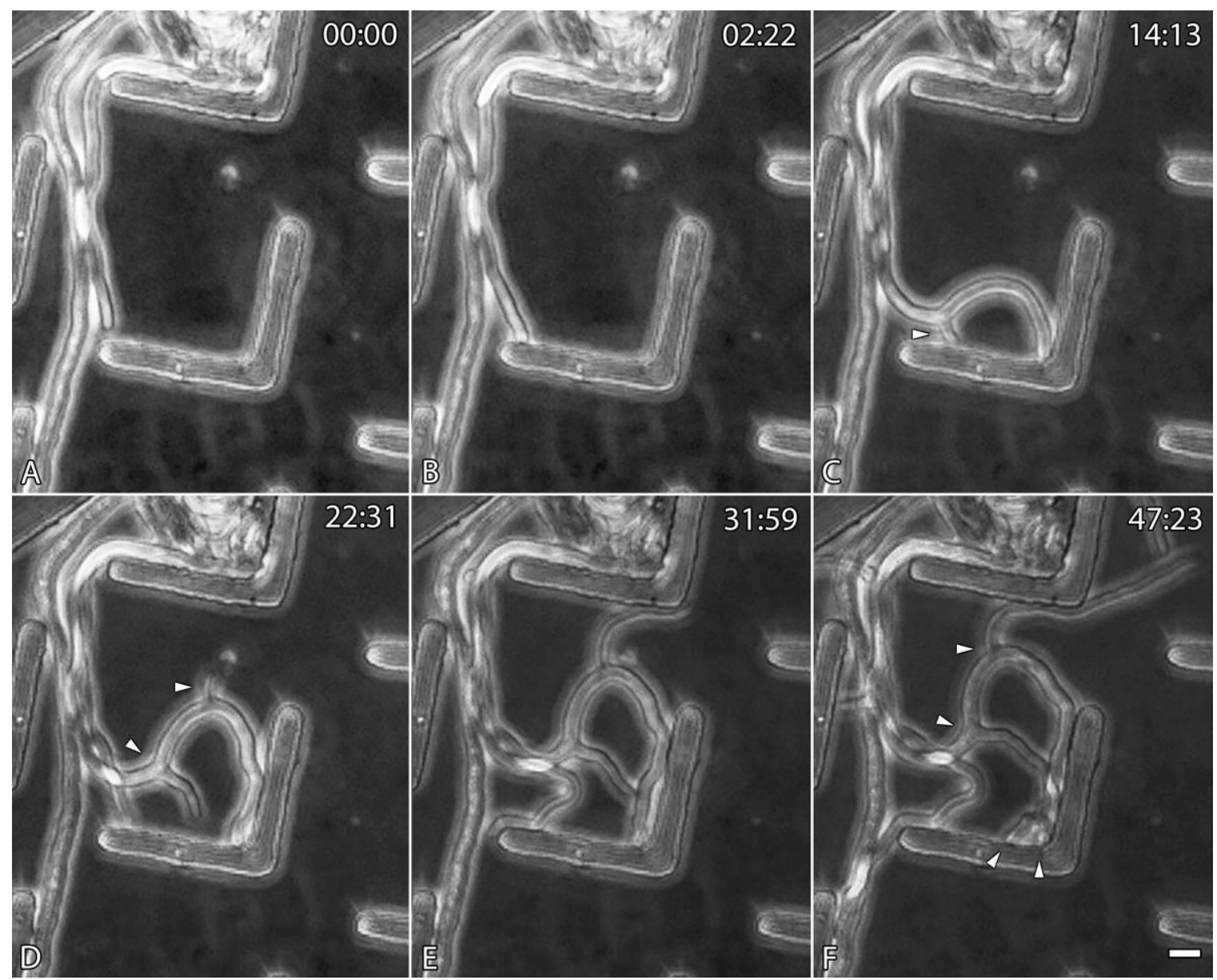

Figure 2. Rhizopus oryzae is seen approaching the edge of a chevron (Fig. A) and then impacting (Fig. B). The hypha continues along the chevron forming a sub-apical branch (Fig. C). A second sub-apical branch forms (Fig. D). The hypha then branches dichotomously (Fig. F). Scale bar $=10 \mu \mathrm{m}$.

\section{References}

[1] J.W. Bennett et al. in 'Cellular and Molecular Biology of Filamentous Fungi' ed. I. K. A. Borkovich, D. J. Ebbole, ASM Press, Washington DC, (2010) pp. 3-7

[2] M. Riquelme et al. Microbiol. Mol. Biol. Rev., 82 (2018) e00068-17

[3] M. Held et al. PNAS, 116 (27) (2019), pp. 13543-13552

[4] H. Wang et al. BMC Evolutionary Biology, 9 (195) (2009)

[5] J.W. Spatafora and B. Robbertse. in 'Cellular and Molecular Biology of Filamentous Fungi' ed. I. K. A. Borkovich, D.J. Ebbole, ASM Press, Washington DC, (2010) pp. 36-49

[6] S.D. Harris. in 'Cellular and Molecular Biology of Filamentous Fungi' ed. I. K. A. Borkovich, D. J. Ebbole, ASM Press, Washington DC, (2012) pp. 238-259

[7] K.E. Fisher and R.W. Roberson. Mycologia, 108 (2016) pp. 533-54 